\title{
Simulation of Spontaneous Heating of a Small Fixed Bed of Dried Coal Exposed to a Flowing Wet Air Stream
}

\section{Appendix: Solving basic equations}

The basic equations were transformed to finite difference equations so as to be solved numerically. Figure A1 shows the grid for formulating the finite difference equations. The solving strategy is to calculate the values of $p, T, q$, and $Q_{\mathrm{j}}$ at the position of $\star \quad(t=t+\Delta t$ and $z=\mathrm{m} \Delta z$ ) from known values shown by . To do so, the finite differential equations are formulated at the position of $\times$. The partial derivatives of $\partial T / \partial t$ and $\partial T / \partial z$ at the position of $\times$ are, for example, given as

$$
\begin{gathered}
\left.\frac{\partial T}{\partial t}\right|_{\text {at x }}=\frac{T_{2}(\mathrm{~m})-T_{1}(\mathrm{~m})}{\Delta t} \\
\left.\frac{\partial T}{\partial z}\right|_{\mathrm{at} \mathrm{x}} \frac{T_{2}(\mathrm{~m})-T_{2}(\mathrm{~m}-1)+T_{1}(\mathrm{~m}+1)-T_{1}(\mathrm{~m})}{2 \Delta z}
\end{gathered}
$$

where subscript 1 and 2 respectively represent the vales at $t=t$ and $t=t+\Delta t$,
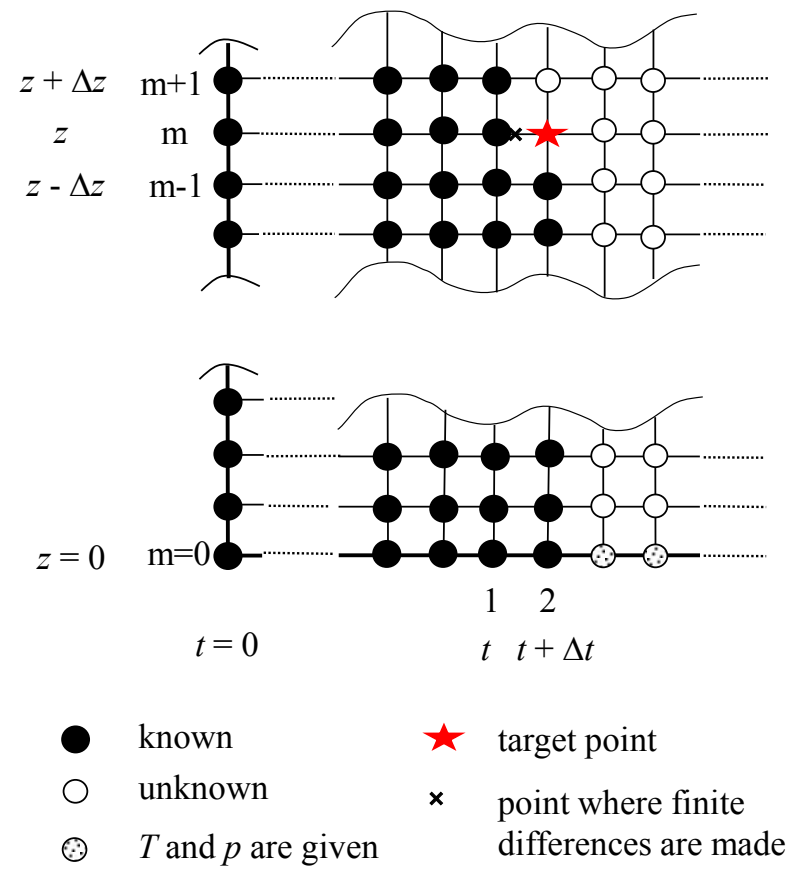

Figure A1. Calculation grid for the simulation. 
Then the basic equations (8) to (10) derived above for the wet air were transformed to finite difference equations as follows:

$$
\begin{aligned}
& \rho_{\mathrm{b}}\left(\frac{q_{2}(\mathrm{~m})-q_{1}(\mathrm{~m})}{\Delta t}+\frac{\varepsilon}{\rho_{\mathrm{b}} R T_{\text {Ave }}} \frac{p_{2}(\mathrm{~m})-p_{1}(\mathrm{~m})}{\Delta t}-\frac{\varepsilon p_{\text {Ave }}}{\rho_{\mathrm{b}} R T_{\text {Ave }}^{2}} \frac{T_{2}(\mathrm{~m})-T_{1}(\mathrm{~m})}{\Delta t}\right) \\
& =-\Phi_{\text {DryAir }} \frac{p_{\mathrm{t}}}{\left(p_{\mathrm{t}}-p_{\mathrm{Ave}}\right)^{2}}\left\{\frac{p_{2}(\mathrm{~m})-p_{2}(\mathrm{~m}-1)+p_{1}(\mathrm{~m}+1)-p_{1}(\mathrm{~m})}{2 \Delta z}\right\} \\
& \rho_{\mathrm{b}} \frac{q_{2}(\mathrm{~m})-q_{1}(\mathrm{~m})}{\Delta t}=\frac{K_{\mathrm{f}} a_{\mathrm{v}}}{R T_{\mathrm{Ave}}}\left(p_{\mathrm{Ave}}-p^{*}{ }_{\mathrm{Ave}}\right) \\
& {\left[\rho_{\mathrm{b}}\left(c_{\mathrm{p}, \text { Coal }}+q C_{\mathrm{p}, \mathrm{H}_{2} \mathrm{O}(\mathrm{Ads})}\right)+\varepsilon\left(\frac{p_{\mathrm{t}}}{R T} C_{\mathrm{p}, \mathrm{Air}}-\frac{p_{\mathrm{t}}-p}{R T^{2}} H_{\mathrm{DryAir}}\right)\right] \frac{T_{2}(\mathrm{~m})-T_{1}(\mathrm{~m})}{\Delta t}+\varepsilon \frac{1}{R T} H_{\mathrm{DryAir}} \frac{p_{2}(\mathrm{~m})-p_{1}(\mathrm{~m})}{\Delta t}} \\
& =-\Phi_{\text {DryAir }} \frac{p_{\mathrm{t}}}{\left(p_{\mathrm{t}}-p_{\mathrm{Ave}}\right)}\left[C_{\mathrm{p}, \mathrm{Air}}\left\{\frac{T_{2}(\mathrm{~m})-T_{2}(\mathrm{~m}-1)+T_{1}(\mathrm{~m}+1)-T_{1}(\mathrm{~m})}{2 \Delta z}\right\}\right] \\
& +\rho_{\mathrm{b}} \Delta H_{\mathrm{Ads}} \frac{q_{2}(\mathrm{~m})-q_{1}(\mathrm{~m})}{\Delta t}+\rho_{\mathrm{b}} \sum_{J-1}^{3} \frac{Q_{\mathrm{j} 2}(\mathrm{~m})-Q_{\mathrm{j} 1}(\mathrm{~m})}{\Delta t}+\Omega_{\mathrm{UA}}\left(T_{\mathrm{s}}-\frac{T_{1}(\mathrm{~m})+T_{2}(\mathrm{~m})}{2}\right)
\end{aligned}
$$

where the heat generation term by the coal-oxygen interaction in Eq. (A5) can be represented as

$$
\frac{Q_{\mathrm{j} 2}(\mathrm{~m})-Q_{\mathrm{j} 1}(\mathrm{~m})}{\Delta t}=\frac{k_{\mathrm{j} 2}\left\{Q_{\mathrm{j} 2}(\mathrm{~m})-Q_{\mathrm{j} 2}(\mathrm{~m})\right\}+k_{\mathrm{j} 1}\left\{Q_{\mathrm{j} 1}(\mathrm{~m})-Q_{\mathrm{j} 1}(\mathrm{~m})\right\}}{2}
$$

All the quantities given with the subscript Ave represent the quantities averaged between $t=t$ and $t=t+\Delta t$ as follows:

$$
T_{\mathrm{Ave}}=\frac{T_{1}(\mathrm{~m})+T_{2}(\mathrm{~m})}{2}, \quad p_{\mathrm{Ave}}=\frac{p_{1}(\mathrm{~m})+p_{2}(\mathrm{~m})}{2}, \quad p_{\mathrm{Ave}}^{*}=\frac{p_{1}^{*}(\mathrm{~m})+p^{*}{ }_{2}(\mathrm{~m})}{2}, \quad q_{\mathrm{Ave}}=\frac{q_{1}(\mathrm{~m})+q_{2}(\mathrm{~m})}{2}
$$

All other quantities which are the functions of $T$ and/or $p$ are all estimated using $T_{\text {Ave }}$ and/or $p_{\text {Ave }}$

Eqs. (A3) to (A5) were then solved numerically with the following boundary/initial conditions:

$$
\begin{aligned}
& T=T_{0}, \quad p \cong p_{\text {Steam } 0} e^{-\frac{K_{\mathrm{r}} a_{\mathrm{V}} S}{v_{0}} z}, q=0 \text { for } t=0 \text { and } z=0 \text { to } z_{\max } \\
& T=T_{0} \text { and } p=p_{\text {Steam } 0} \text { for } \quad z=0 \text { and } t=0 \text { to } t_{\max }
\end{aligned}
$$

where $p_{\text {Steam } 0}$ is the vapor pressure of the inlet gas stream and $v_{0}$ is the volumetric flow rate of wet air at the inlet conditions. 